\title{
Red de sensores de humedad y temperatura de bajo costo
}

\section{Low cost temperature and humidity sensor network}

MENDOZA-PINTO, Luis Manuel, ESPINOSA-TRUJILlO, María Jesús y PEET-MANZÓN, Jesús Humberto

Universidad Tecnológica Metropolitana

ID $1^{\text {er }}$ Autor: Luis Manuel, Mendoza-Pinto / ORC ID: 0000-0002-0786-2602, Researcher ID Thomson: T-3628-2018, CVU CONACYT ID: 458937

ID $1^{\mathrm{er}}$ Coautor: María Jesús, Espinosa-Trujillo / ORC ID: 0000-0002-2889-4849, Researcher ID Thomson: T-3632-2018, CVU CONACYT ID: 297283

ID $2^{\text {do }}$ Coautor: Jesús Humberto, Peet-Manzón / ORC ID: 0000-0002-4807-5349, CVU CONACYT ID: 1002109

DOI: $10.35429 /$ JEA.2019.18.6.11.18

Recibido: 19 de Enero, 2019; Aceptado 02 de Marzo, 2019

\section{Resumen}

En este artículo se presenta el desarrollo de una red de bajo costo de sensores inalámbricos que utilizan una plataforma de hardware de código abierto, compuesta por un ESP8266 y un sensor digital de temperatura-humedad para medir los parámetros en un área determinada por el rango del sensor. El desarrollo general del sistema incluye el uso de un software de código abierto para recibir la información a través de la red. Se realizaron pruebas de la efectividad del sensor en tres puntos diferentes de un área con aire acondicionado. El primer sensor se colocó en el exterior del área, el segundo en medio y el último a la salida del aire acondicionado. Los resultados obtenidos permitieron conocer el comportamiento de la temperatura y humedad en el área y la efectividad de la red de sensores para medir las variables, los resultados de las mediciones se presentan en detalle. Debido a que el sistema es altamente escalable, de bajo costo y fácil de construir en comparación con otros sistemas, es una buena opción para una amplia variedad de aplicaciones.

Red de sensores, ESP8266, Medición

\begin{abstract}
This article presents the development of a low cost network of wireless sensors that use an open source hardware platform, consisting of an ESP8266 and a digital temperature-humidity sensor to measure the parameters in an area determined by the range of the sensor. The general development of the system includes the use of open source software to receive information through the network. Tests of sensor effectiveness were performed at three different points in an air-conditioned area. The first sensor was placed outside the area, the second in the middle and the last one at the exit of the air conditioning. The results obtained allowed to know the behavior of temperature and humidity in the area and the effectiveness of the sensor network to measure the variables, the results of the measurements are presented in detail. Because the system is highly scalable, inexpensive and easy to build compared to other systems, it is a good choice for a wide variety of applications.
\end{abstract}

Sensor network, ESP8266, Measurement

Citación: MENDOZA-PINTO, Luis Manuel, ESPINOSA-TRUJILLO, María Jesús y PEET-MANZÓN, Jesús Humberto. Red de sensores de humedad y temperatura de bajo costo. Revista de Aplicaciones de la Ingeniería. 2019 6-18: 11-18

\footnotetext{
* Correspondencia del Autor (Correo electrónico: maria.espinosa@utmetropolitana.edu.mx)

$\dagger$ Investigador contribuyendo como primer autor.
} 


\section{Introducción}

Se han realizado diversos trabajos en el desarrollo de redes inalámbricas ya que la medición, vigilancia y control de los diferentes eventos en nuestra vida diaria siempre están presentes. En este contexto, podemos ver los trabajos realizados por Thomanek, et al., (2011), donde el uso de filtros FIR (Finite impulse Response) y cámaras para detectar peatones en los cruceros de tránsito se vuelve un tema de alta importancia. Así mismo Kanchumarthy \& Viswanathan, (2006), realizaron un trabajo sobre el impacto de los sensores inalámbricos centrados en el rendimiento de la detección, probando diferentes sensores bajo diferentes ambientes a fin de comprobar la interferencia, desconexión y resultado en el uso de sensores de fusión (multi-sensores).

Sung y Tsai, (2011), desarrollaron una plataforma con ZigBee CC2530 aplicada a varios tipos de sensores específicos para sistemas de monitoreo ambiental cuyo objetivo es mejorar la agregación de señales inalámbricas multi-sensor mediante la fusión de decisiones de varios bits. ZigBee es un estándar de transmisión inalámbrica de corto alcance basado en IEEE 802.15.4, formulado por el protocolo ZigBee Alliance. Es de bajo costo, bajo consumo de energía y transmisión de corta distancia a una velocidad de transmisión de 250 kbps para redes de sensores inalámbricos. Sus principales aplicaciones incluyen temperatura, humedad y otros tipos de monitoreo de datos, automatización de fábrica, domótica, monitoreo remoto y control de dispositivos domésticos.

Yilmaz, (2007), analizó algunas de las técnicas de fusión de sensores más comunes. Aunque no se da prioridad a una técnica sobre las otras, se seleccionaron sólo un puñado de técnicas que están relacionadas con la fusión de sensores en el contexto del modelado de ciudades urbanas.

Tomovic et al., (2015), reportaron las limitaciones de recursos de los nodos sensores como una debilidad de las redes de sensores inalámbricas (WSN). En su trabajo proponen una arquitectura WSN basada en principios de red definida por software (SDN), que permite la reconfiguración automática de la red y la gestión óptima de los nodos sensores con limitaciones de energía con capacidades de comunicación limitadas.
Los trabajos realizados por Bharti et al., (2017), mencionan el crecimiento exponencial de los recursos en Internet-of-Things (IoT), lo cual se ha convertido en uno de los principales desafíos debido a la necesidad de recursos de autogestión. Proponen un "Marco de Inquisición de Recursos Inteligentes en Internet de las Cosas (IRIF-IoT)" que aborde los desafíos a través de tres capas, a saber, la percepción, el descubrimiento y la aplicación.

Hadwan y Reddy, (2016), presentan un sistema de control y monitoreo doméstico, flexible y económico, utilizando una red basada en Raspberry Pi y utilizando el microcontrolador Arduino. La interfaz entre el punto de acceso y los conmutadores con conectividad IP para acceder a dispositivos y controlarlos de forma remota, se realiza mediante una aplicación para teléfonos inteligentes basada en Android o una computadora servidor. El hardware principal de este sistema contiene: Raspberry Pi, microcontrolador arduino, transceptor inalámbrico nRF24L01, módulos de relé, interruptores, lámparas, enchufes, sensores y juego de cables. El Resultado de este sistema fue la programación mediante Python que viene incorporado con Raspbian, y el uso del adaptador de módulo inalámbrico para realizar conexiones entre el microcontrolador arduino y el transceptor inalámbrico nRF24L01.

Ungurean y Brezulianu, (2017), proponen una arquitectura de IoT que se puede usar en la atención médica para monitorear las señales de ECG (electrocardiograma) adquiridas de forma independiente por el paciente, utilizando un teleelectrocardiógrafo móvil, sin la ayuda de un especialista. En su trabajo describen las características principales del dispositivo móvil y cómo se integran estas características en la arquitectura de IoT que proponen.

Por otro lado, Barbosa et al., (2019), proponen un red de sensores inalámbricos para el monitoreo de variables agroecológicas, basados en un ESP8266 con sensores de temperatura y humedad, DS18B20 y DTH-11 respectivamente. En este trabajo utilizan una plataforma de IoT thingspeak y la app inventor para realizar la conexión con bluetooth.

Luna et al., (2018), realizaron una red de sensores para calcular el balance energético en un invernadero. La red consta de una Raspberry Pi y sensores DS18B20 y DTH-11. 
Manjarrez et al., (2016), diseñaron un dispositivo para monitoreo de temperatura y control de humedad para plantas, utilizando un microcontrolador ESP8266, Arduino nano, sensores YL-69, YL-38 y DTH-11. El monitoreo se realiza por medio de una aplicación desarrollada en Android y para el control utilizan un modelo de red neuronal llama Hyperconic Multilayer Perceptron.

Basado en los trabajos anteriores y sus diferentes aplicaciones, se plantea la elaboración de un sistema de sensores de temperatura que ayude al monitoreo de temperatura y humedad en casas habitación, centros comerciales e industrias que estén interesados en que se pueda llevar a cabo un monitoreo a distancia, a fin de poder procesar la información para la toma de decisiones en el mantenimiento y remplazo de equipos de aire acondicionado y climatización.

\section{Desarrollo}

Se plantea la implementación de una red de sensores "Sensor Network", utilizando dispositivos que permiten obtener lecturas de temperatura con un formato de salida digital DHT-11, conocidos como smartsensors. Como primera opción se utilizó un microcontralador tipo Arduino UNO para la adquisición de los datos del sensor, debido a que la tarjeta de Ardunio no posee un dispositivo integrado para la comunicación WiFi, se tuvo que adicionar un dispositivo periférico para obtener la función deseada y posteriormente enviar los datos vía puerto serial a una computadora para almacenarlos y posteriormente analizarlos, presentarlos en forma gráfica y enviarlos a través de internet a otros dispositivos. Este primer sistema se puede ver en la Figura 1.

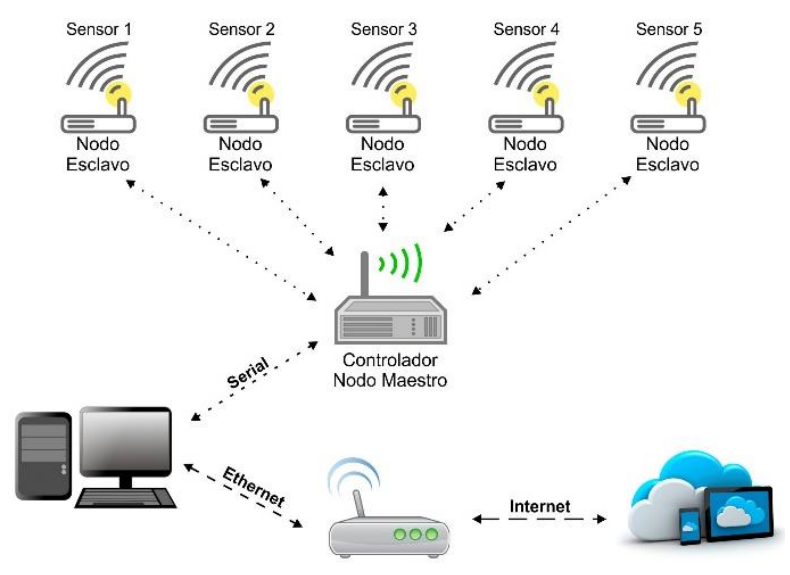

Figura 1 Red de Sensores con Controlador Maestro Esclavo y comunicación serial para transmitir los datos Fuente: Elaboración Propia
Como se ve en la Figura 1, tener un dispositivo maestro donde todos los sensores se conectan a través de un host y que sirve de puente con el equipo de cómputo, es poco eficiente. Actualmente se pueden utilizar las tecnologías disponibles para conectar directamente la computadora y los sensores a la red local, por lo que no es necesario un intermediario que recopile la información, solo sería válido si se desean guardar los datos en un punto específico, o no hay un equipo de cómputo disponible dedicado para la recolección de estos.

Debido a lo anterior, se opta por utilizar un microcontrolador ESP8266 que permite la comunicación de los datos a través de una red tipo Wi-Fi. Este microcontrolador proporciona prestaciones como procesador $\mathrm{y}$, por lo tanto, se utilizará como unidad de procesamiento para capturar la información del sensor y enviar los datos a través de la red. Este modelo de conexión se puede observar en la Figura 2, en este modelo se cambia el nodo maestro por una computadora que sirve como Servidor Web, la cual permitirá acceder a los datos y realizar el análisis de estos.

La programación de un Servidor Web no es muy complicada de realizar, sin embargo, para hacer un proceso más eficiente y hacer uso de herramientas tecnológicas disponibles, se decide utilizar una aplicación de código libre que, no sólo recolecta la información para monitorearla, sino que además puede obtener el control sobre diversos dispositivos Wi-Fi.

El propósito principal de este software es el control de dispositivos inteligentes, diseñado para la domótica residencial.

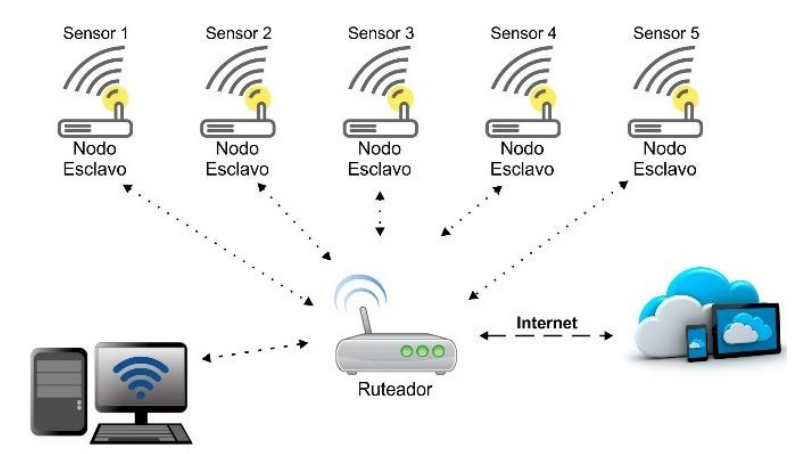

Figura 2 Red de sensores conectados a la intranet y enviando información a una Computadora Fuente: Elaboración Propia 
El programa descrito anteriormente es DOMOTICZ @ , el cual puede realizar el control de diferentes dispositivos de tecnologías comerciales disponibles $\mathrm{y}$, además, tiene un apartado para poder adicionar dispositivos de monitoreo y control personalizados que operen en la red local. Esta fue la razón por la cual se decidió que fuera el programa de monitoreo de los sensores, adicionalmente permite guardar y graficar los datos.

\section{Construcción de los dispositivos de monitoreo.}

Se opta por utilizar una tarjeta basada en el microcontrolador ESP8266, cuyo desarrollo es compatible con el modelo comercial WeMos D1 Mini, el cual se muestra en Figura 3.

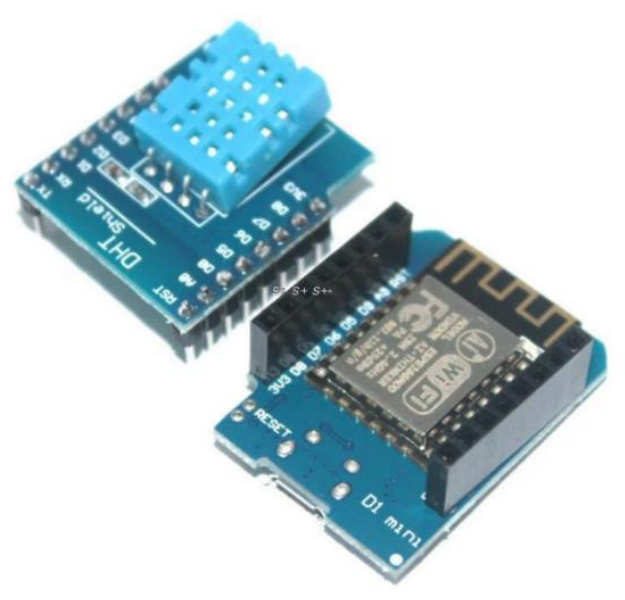

Figura 3 Tarjeta de desarrollo ESP8266 tipo WeMos D1 Mini genérica y sensor digital DHT-11 en una placa diseñada para la misma tarjeta de desarrollo Fuente: https://www.newegg.com/p/2S7-00MH-02Y35

La programación del microcontrolador se efectua a través de la IDE de Arduino, el IDE puede ser utilizado para programar estos microcontroladores con una pequeña configuración. La placa del sensor ya está preparada para que los pines concidan con los de la tarjeta de desarrollo. Las características de estos componentes se describen en las siguientes secciones.

\section{Funcionamiento del DHT-11.}

El DHT-11 utiliza una comunicación simplificada de un sólo bus con una sola línea de datos que funciona como el sistema de intercambio de datos y también es controlado por este único bus.
Este dispositivo conectado a través de un puerto de drenaje abierto o tri-estado permite que el dispositivo no envíe datos para liberar el bus, mientras que otros dispositivos usan el bus. Un solo bus requiere por lo general una resistencia externa de $5.1 \mathrm{k} \Omega$ para el estado alto, de modo que cuando el bus esté inactivo su estado sea alto.

\begin{tabular}{|l|l|}
\hline \multicolumn{2}{|c|}{ Característica } \\
\hline $\begin{array}{l}\text { Resolución de humedad } \\
\text { relativa: }\end{array}$ & 16 Bits \\
\hline Repetibilidad: & $\pm 1 \% \mathrm{RH}$ \\
\hline Precisión: & a $25^{\circ} \mathrm{C} \pm 5 \%$ \\
\hline Intercambiabilidad de HR: & totalmente intercambiable \\
\hline Tiempo de respuesta: & $\begin{array}{l}1 / \mathrm{e}(63 \%) \text { de } 25^{\circ} \mathrm{C} 6 \mathrm{~s} 1 \mathrm{~m} \\
/ \mathrm{s} \text { aire } 6 \mathrm{~s}\end{array}$ \\
\hline Histéresis: & $< \pm 0.3 \%$ \\
\hline HR Estabilidad a largo plazo: & $< \pm 0.5 \% \mathrm{RH} / \mathrm{yr}$ in \\
\hline Resolución de temperatura: & 16 bits \\
\hline Repetibilidad: & \pm 0.2 \\
\hline Rango: & a $25^{\circ} \mathrm{C} \pm 2$ time \\
\hline Tiempo de respuesta: & $1 /$ e $(63 \%) 10 \mathrm{~S}$ \\
\hline Fuente de alimentación: & DC $3.5 \sim 5.5 \mathrm{~V}$ \\
\hline Corriente de suministro: & $\begin{array}{l}\text { en medición } 0.3 \mathrm{~mA}, \text { en } \\
\text { espera } 60 \mu \mathrm{A}\end{array}$ \\
\hline Período de muestreo: & más de 2 segundos. \\
\hline
\end{tabular}

Tabla 1 Especificaciones Técnicas DHT - 11

Fuente: (Aosong(Guangzhou) Electronics Co., 2007)
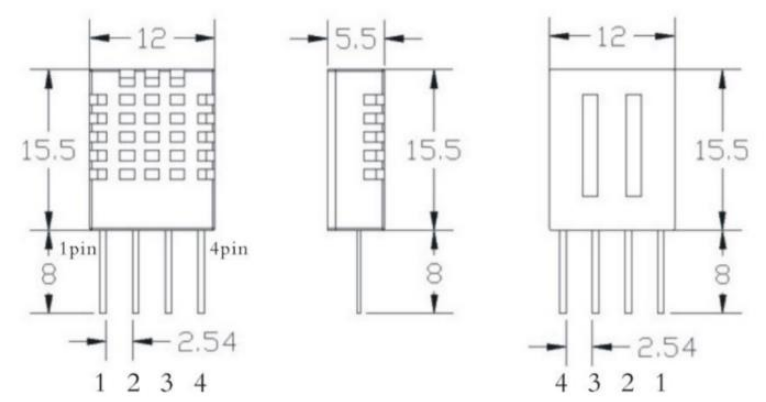

Figura 4 Dimensiones del sensor DHT-11

(Aosong(Guangzhou) Electronics Co., 2007)

Debido a que la estructura es maestro esclavo, y sólo cuando el anfitrión llama al esclavo, el esclavo responde, los dispositivos de acceso del anfitrión deben seguir estrictamente la secuencia de un solo bus, si la secuencia es caótica, el dispositivo no responderá al anfitrión. (Aosong (Guangzhou) Electronics Co., 2007).

Las características técnicas se muestran en la Tabla 1, donde se puede observar que la resolución del sensor es de 16 bits. El tamaño del sensor también es convenientemente pequeño como se puede observar en la Figura 4. 


\section{WeMos D1 Tarjeta de desarrollo.}

La tarjeta de desarrollo WeMos D1 mini pro tiene las características técnicas descritas en la Tabla 2, y la configuración de las conexiones en la placa se deben de realizar de acuerdo con la Figura 5.

\begin{tabular}{|l|r|}
\hline \multicolumn{1}{|c|}{ Característica } & Especificación /Valor \\
\hline Microcontrolador & ESP-8266EX \\
\hline Tensión de funcionamiento & $3.3 \mathrm{~V}$ \\
\hline E/S Digitales & 11 \\
\hline Entradas analógicas & $1($ Max input: $3.2 \mathrm{~V})$ \\
\hline Velocidad de reloj & $80 \mathrm{MHz} / 160 \mathrm{MHz}$ \\
\hline Destello & $16 \mathrm{M}$ bytes \\
\hline Longitud & $34.2 \mathrm{~mm}$ \\
\hline Anchura & $25.6 \mathrm{~mm}$ \\
\hline Peso & $2.5 \mathrm{~g}$ \\
\hline
\end{tabular}

Tabla 2 Características Técnicas de la tarjeta de desarrollo WeMos

Fuente: $\quad$ https://einstronic.com/store/internet-ofthings/esp8266/wemos/wemos-dl-mini-pro-esp8266-wifidevelopment-board/

\section{Microcontrolador ESP8266EX}

La Plataforma de Conectividad Inteligente (ESCP) de Espressif Systems es un conjunto de SOCs inalámbricos de alto rendimiento, de alta integración, desarrollados para diseñadores de plataformas móviles con limitación de espacio y potencia. Esta plataforma incorpora capacidades Wi-Fi dentro de otros sistemas y también puede funcionar como una aplicación independiente, con el menor costo y un mínimo espacio requerido.

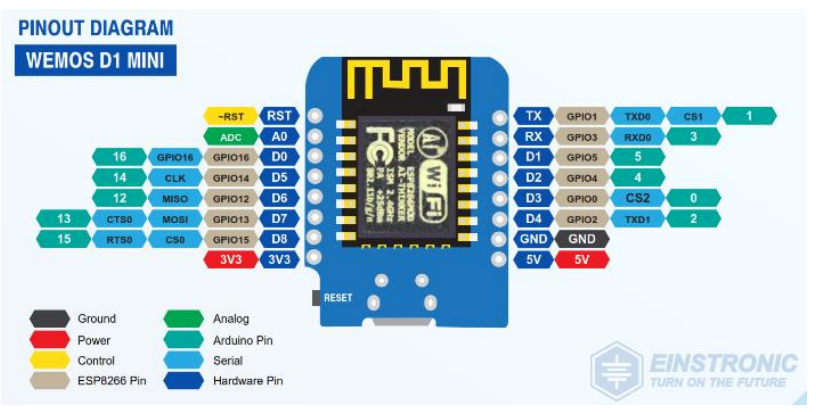

Figura 5 Asignación de Pines tarjeta de desarrollo compatible WeMos D1 mini (Einstronic.com, 2017)

El ESP8266EX ofrece una solución de red Wi-Fi completa y autónoma; puede utilizarse para alojar una aplicación o para activar funciones de red Wi-Fi desde otro procesador de aplicaciones. Cuando el ESP8266EX aloja la aplicación, ésta se inicia directamente desde una memoría flash externa. Posee integrado un caché para mejorar el rendimiento del sistema en dichas aplicaciones.
Alternativamente, como adaptador Wi-Fi, el acceso inalámbrico a Internet se puede agregar a cualquier diseño basado en microcontroladores con una conectividad simple (interfaz SPI / SDIO o I ${ }^{2} \mathrm{C} / \mathrm{UART}$ ).

El ESP8266EX es uno de los chips Wi-Fi con mayor grado de integración de la industria; ya que incluye dispositivos como los conmutadores de antena, el balun RF, el amplificador de potencia, el amplificador de recepción de bajo ruido, los filtros, los módulos de administración de energía, requiere un mínimo de circuitos externos y todo el conjunto, incluido el módulo frontal, está diseñada para ocupar un área mínima en un PCB.

El ESP8266EX también integra una versión mejorada del procesador de 32 bits de Tensilica L106 Diamond, con SRAM incorporada, además de las funcionalidades WiFi. ESP8266EX a menudo está integrado con sensores externos y otros dispositivos específicos de aplicación a través de sus GPIOs. (Espressif Systems, 2018)

Por todas las prestaciones mencionadas anteriormente, se decidió utilizar dicho microcontrolador y el sensor de temperatura DHT-11 para construir el sensor inteligente. Para complementar la construcción se utilizó una batería recargable tipo banco de energía, de 2200 mAh y 5 VCD, como fuente de alimentación independiente. Esta configuración permite la movilidad del sensor dónde se necesite recabar los datos. Ver Figuras 6 y 7.

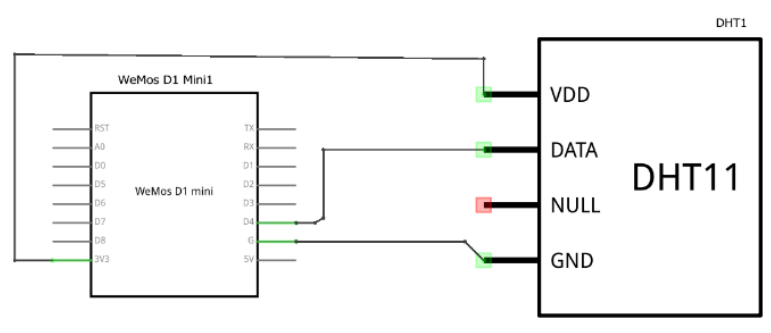

fritzing

Figura 6 Diagrama de conexión de la tarjeta de desarrollo WeMos D1 mini y el sensor de temperatura y humedad Fuente: Elaboración Propia 


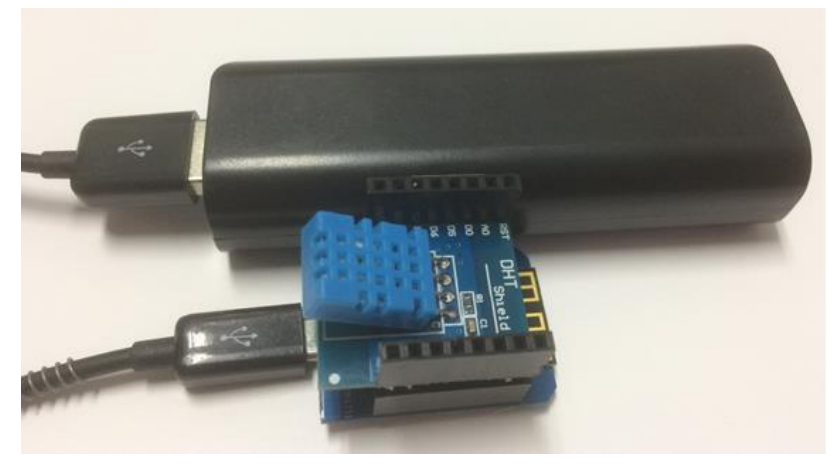

Figura 7 Sistema WeMos + DHT - 11 + Alimentación de $2200 \mathrm{mAh}$

Fuente: Elaboración Propia

Posterior a la construcción se desarrolla el código de programación para el hardware, con el propósito de proveer la información al Servidor Web. Se construyeron tres sensores para obtener diferentes datos, a fin de comparar la información recolectada.

Para el desarrollo del software primero se establecen los parámetros de configuración del hardware a través de librerias desarrolladas por terceros.

\#include <ESP8266WiFi.h>

\#include <ESP8266HTTPClient.h>

\#include <DHT.h>

\section{\#define DHTTIPO DHT11 \\ \#define DHTPIN D4}

También se deben definir los parámetros que permitan acceder a la red. En este caso se utilizan IP fijas para cada sensor, y se debe establecer el SSID de la red, el password y la IP del host que contiene el servidor web.

const char* ssid = "SSIDRed";

const char* password = "password_WIFI";

const char* host $=$ "XXX.XXX.XX.XXX";

Por último, se definen el puerto por el cual se va a acceder al servidor y el index, o identificador del sensor. Así también se inicializa el sensor y el cliente HTTP.

const int idx $=3$;

const int port $=8080$;

DHT dht(DHTPIN, DHTTIPO);

HTTPClient http;

En el setup se inicializa, de forma opcional, el puerto serial a los bps que se desea comunicar con un equipo de cómputo, la intención de esto es asegurar que el dispositivo se conecta correctamente con el router para enviar, a través del UART, el estado del wifi y detectar si la conexión se ha podido realizar, adicionalmente se puede utilizar para determinar si se ha enviado la información, así como si el servidor la ha recibido correctamente.

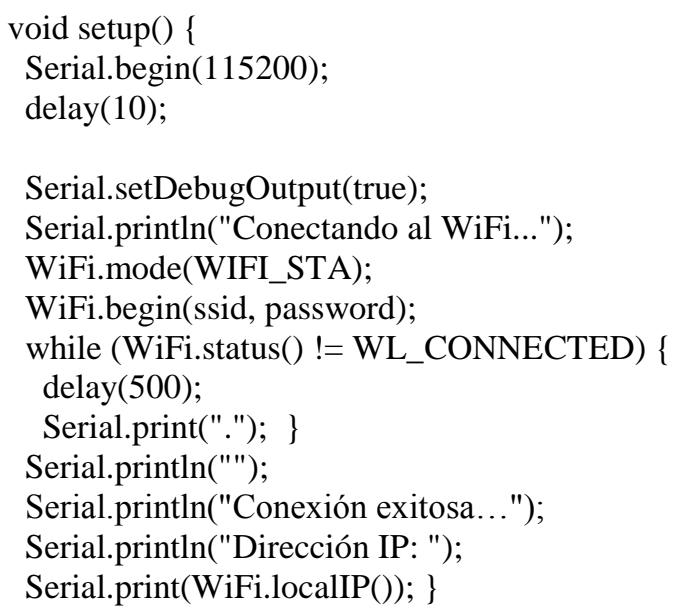

Serial.setDebugOutput(true);

Serial.println("Conectando al WiFi...");

WiFi.mode(WIFI_STA);

WiFi.begin(ssid, password);

while (WiFi.status() != WL_CONNECTED) \{

delay (500);

Serial.print("."); \}

Serial.println("");

Serial.println("Conexión exitosa...");

Serial.println("Dirección IP: ");

Serial.print(WiFi.localIP()); \}

El programa principal realiza dos funciones, en primer lugar leer los datos del sensor y, posteriormente, enviarlos a través de la conexión WiFi cada determinado tiempo. Para nuestras pruebas se seleccionó un intervalo de 5 segundos.

\section{void loop() \{}

unsigned long currentMillis = millis $($ );

if ( currentMillis - previousMillis > watchdog ) \{

previousMillis $=$ currentMillis;

if(WiFi.status() != WL_CONNECTED) \{

Serial.println("Conexión al WiFi no existente !");

\} else \{

Serial.println("Enviando datos al servidor...");

float $\mathrm{t}=$ dht.readTemperature();

float $\mathrm{h}=$ dht.readHumidity () ;

if $(\operatorname{isnan}(\mathrm{t}) \|$ isnan(h) ) \{

Serial.println("DHT ok");

\} else \{

int hum_stat;

int bar_for $=0$;

if $(h>70)\{$

hum_stat $=3$;

\} else if $(h<30)\{$

hum_stat $=2$;

\} else if $(\mathrm{h}>=30 \& \mathrm{~h}<=45)\{$

hum_stat $=0$;

\} else if $(h>45 \& h<=70)\{$

hum_stat $=1$;

\}

String url = "http://";

url += host;

url += ":";

url += String(port);

url

"/json.htm?type=command\&param=udevice \&idx=";

url $+=$ String (idx);

url += "\&nvalue=0\&svalue=";

url += String $(\mathrm{t}) ;$ url += ";";

url += String(h); url += ";";

url += String(hum_stat); url += ";";

sendData(url);

\}\}\}\}

void sendData(String url) \{

Serial.print("conectando... ");

Serial.println(host);

Serial.print("Solicitando URL: ");

Serial.println(url);

http.begin(url);

int httpCode $=$ http.GET();

MENDOZA-PINTO, Luis Manuel, ESPINOSA-TRUJILLO, María Jesús y PEET-MANZÓN, Jesús Humberto. Red de sensores de humedad y temperatura de bajo costo. Revista de Aplicaciones de la Ingeniería. 2019. 
Serial.println(httpCode);

if (httpCode) \{

if (httpCode $==200)$ \{

String payload $=$ http.getString () ;

Serial.println("Respuesta del servidor");

Serial.println(payload); \}\}

Serial.println("cerrando conexión...");

http.end();\}

Como el software seleccionado para recibir los datos ya ha sido creado y es de código abierto, es importante la forma en que se enviarán los datos, para esto se realiza la construcción de un comando JSON (JavaScript Object Notation) que es un formato ligero de intercambio de datos. Este formato es fácil de leer y escribir para una persona y, así mismo, es fácil de analizar y generar para las máquinas. Se basa en un subconjunto del lenguaje de programación de JavaScript, Estándar ECMA$262,3^{\text {a }}$ edición, diciembre de 1999 . JSON es un formato de texto que es completamente independiente del lenguaje, pero utiliza convenciones que son familiares para los programadores de la familia $\mathrm{C}$ de lenguajes, incluida la C, C ++, C \#, Java, JavaScript, Perl, Python y muchos otros. Estas propiedades hacen de JSON un lenguaje ideal para el intercambio de datos. (Ecma International, 2014)

Los comandos JSON permiten generar y analizar los objetos de lenguaje de formato JSON. Especificamente, el formato JSON hace que sea posible el acceso a las bases 4D (datos y estructura) utilizando un navegador web.

Una vez que el hardaware es capaz de enviar datos, es necesario instalar y configurar el software DOMOTICZC para que pueda recibir la información y desplegarla en pantalla. Esta configuración se puede observar en la Figura 8.

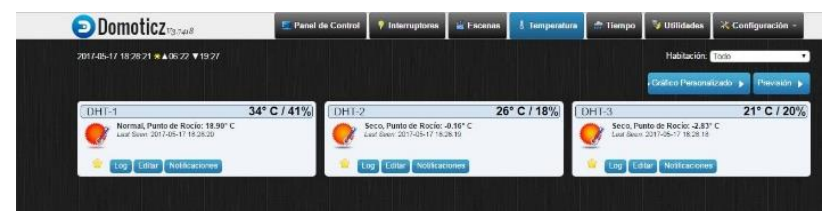

Figura 8 Sensores operando en el programa DOMOTICZ@

Fuente: Elaboración Propia

\section{Pruebas de funcionamiento}

Las pruebas de funcionamiento se realizaron utilizando tres sensores, a fin de que se puedan realizar mediciones en diferentes puntos.
Los sensores fueron colocados, uno en la intemperie, otro en una locación cerrada climatizada y, el tercero, en el punto de salida del aire acondicionado $(\mathrm{A} / \mathrm{C})$.

\section{Resultados}

Se realizó la medición en tres puntos de una oficina de una institución educativa para obtener los datos de temperatura y humedad. El primer sensor se colocó en el exterior de la oficina, los otros dos se colocaron de forma que el primero estuviera alejado del A/C y el segundo se colocó a la salida del A/C.

Los resultados obtenidos se muestran en la Figura 9 (gráfica de temperaturas) y en la Figura 10 (gráfica de porcentajes de humedad). Se pudo observar que los sensores trabajaron de forma bastante estable, el envío de datos fue continuo y en ningún momento se dejó de recibir lecturas, esto se debe a que la red conformada por el router tenía solo el tráfico de los sensores.

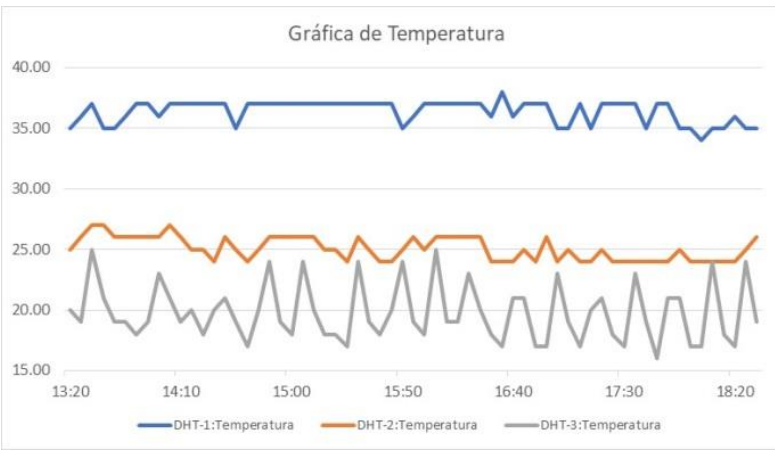

Figura 9 Gráfica de temperaturas Fuente: Elaboración Propia

Los sensores proporcionaron los datos solicitados y se pudo apreciar la efectividad de usar una red Wi-Fi para la transmisión de datos, coincidiendo con el estudio realizado por Wang y Chi (2016).

En la gráfica de temperaturas se puede observar la línea gris correspondiente al DHT 3 mostrando picos, estos representan el rango de actuación del sensor del A/C y, por consiguiente, el arranque y paro del compresor del A/C, mientras que el DHT -2 representa la temperatura del cuarto en el que se llevó a cabo la medición. Se puede observar la funcionalidad del A/C para mantener la temperatura de la habitación al compararla con la temperatura exterior indicada por medio de la línea azul, correspondiente al DHT -1 . 


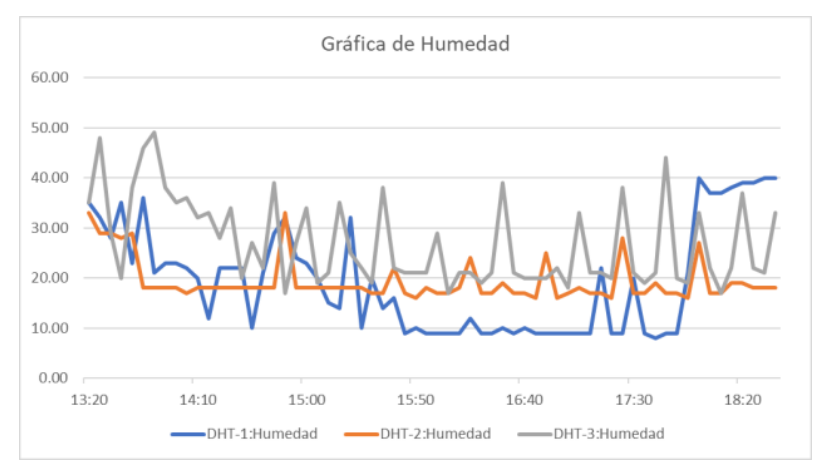

Figura 10 Gráfica de porcentajes de humedad Fuente: Elaboración Propia

Con respecto a la humedad se puede observar que se conservó casi constante durante el transcurso de la tarde, durante la mañana descendió y por la tarde/ noche empezó a aumentar nuevamente.

\section{Conclusiones}

Se puede concluir que la implementación de la Sensor Network se ha logrado, fue posible detectar la temperatura y humedad desde diversos puntos en el sitio de pruebas.

El uso de estos sensores es relativamente sencillo, de bajo costo y resultan muy eficaces para medir diferentes parámetros, es importante mencionar que las capacidades del circuito y la tarjeta van más allá de sólo ser un sensor (Sinha et al. 2015). Con la construcción propuesta se puede implementar el uso de otro tipo de sensores e inclusive controlar el encendido o apagado de éstos, por medio de relevadores u otros sistemas de control.

La implementación de este sensor para monitoreo de los sistemas de $\mathrm{A} / \mathrm{C}$ en locaciones lejanas puede permitir que se tenga, además de un historial, los datos estadísticos que permitan predecir una falla en el A/C. Cuando se detecta que la diferencia entre la temperatura de la habitación y la ambiental es menor a un rango determinado y los picos de arranque son más frecuentes, esto puede ser indicativo de que el sistema de refrigeración está fallando (Hernández, 2009).

Como continuación de los trabajos realizados se plantea el uso de sensores para llevar a cabo la medición de la corriente y voltaje, así como del respaldo de los datos en tarjetas SD. También se puede implementar alertas para el sistema de batería baja y una función para la calibración de los sensores.
Otra posibilidad es el uso de modelos de ultrabajo consumo de energía, al utilizar funciones en modo de suspensión en el código del WeMos. Por otro lado, también se puede desarrollar, en Java Script o Html, una interfaz Web personalizada y usar en tiempo real los comandos JSON y las bases de datos para ampliar el rango de aplicación de los sensores, al no limitarlos a una red de acceso local.

\section{Referencias}

Aosong(Guangzhou) Electronics Co., L. (2007). DHT-11 Datasheet (p. 4). p. 4. Retrieved from http://www.aosong.com/en/products/details.asp ?id=109

Barbosa Pira, D. D., Sanabria Rodríguez, J. S., Bueno Mesa, H. C., \& Edgar Aguirre, D. V. (2019). Red de sensores inalámbricos para el monitoreo de variables agroecológicas en cultivos bajo invernadero. Retrieved October 4, 2019, from Revista Publicaciones e Investigación website: http://hemeroteca.unad.edu.co/index.php/public aciones-e-investigacion/article/view/2781/3260

Bharti, M., Saxena, S., \& Kumar, R. (2017). Intelligent Resource Inquisition Framework on Internet-of-Things. Computers and Electrical Engineering, 58, 265-281. https://doi.org/10.1016/j.compeleceng.2016.12. 023

Ecma International. (2014). The JavaScript Object Notation (JSON) Data Interchange Format. In T. Bray (Ed.), Ecma International. https://doi.org/10.17487/rfc7158

Einstronic.com. (2017). WeMos Series, Mini.

Espressif Systems. (2018). Esp8266Ex. Retrieved from https://espressif.com/en/products/hardware/esp8 266ex/overview

Hadwan, H. H., \& Reddy, Y. P. (2016). Smart home control by using Raspberry Pi \& Arduino UNO. International Journal of Advanced Research in Computer and Communication Engineering, 5(4), 283-288. https://doi.org/10.17148/IJARCCE.2016.5473

Hernández Valadez, J. (2009). Manual de Refrigeración Doméstica (3a ed.). Trillas. 
Kanchumarthy, V., \& Viswanathan, R. (2006). Further Results on the Impact of Quality of Wireless Sensor Links on Decentralized Detection Performance. 2006 40th Annual Conference on Information Sciences and Systems, (April), 44-49. https://doi.org/10.1109/CISS.2006.286428

Luna Arauz, S. E., Vallejo Andrade, A. A., \& Rubiñoz Panta, J. E. (2018). Supervisión de temperatura y humedad para el cálculo de balance energético en un invernadero con tiempos de muestreo obtenidos de forma experimental. Pistas Educativas, 39(128), 1014$1027 . \quad$ Retrieved from http://itcelaya.edu.mx/ojs/index.php/pistas

Manjarrez Carrillo, Ander O. Chávez, Banda, J. M., Damián Padilla, L. Y., Vidal, Rodríguez, L. M., Peraza Arjona, O. F., Herrera Guzmán, R., \& Serrano Rubio, J. P. (2016). Diseño de un dispositivo para monitoreo de temperatura y control de humedad de plantas. Pistas Educativas, (120), 832-847.

Sinha, N., Pujitha, K. E., \& Alex, J. S. R. (2015). Xively based sensing and monitoring system for IoT. 2015 International Conference on Computer Communication and Informatics (ICCCI), $1-6$. https://doi.org/10.1109/ICCCI.2015.7218144

Sung, W. T., \& Tsai, M. H. (2011). Multi-sensor wireless signal aggregation for environmental: Monitoring system via multi-bit data fusion. Applied Mathematics and Information Sciences, 5(3), 589-603.

Thomanek, J., Ritter, M., Lietz, H., \& Wanielik, G. (2011). Comparing Visual Data Fusion Techniques Using FIR and Visible Light Sensors to Improve Pedestrian Detection. 2011 International Conference on Digital Image Computing: Techniques and Applications, (December), 119-125. https://doi.org/10.1109/DICTA.2011.27

Tomovic, S., Radonjic, M., Pejanovic-djurisic, M., \& Radusinovic, I. (2015). Software-Defined Wireless Sensor Networks: opportunities And Challenges. ETF Journal of Electrical Engineering, 21(1), 75-83.
Ungurean, L., \& Brezulianu, A. (2017). An internet of things framework for remote monitoring of the healthcare parameters. Advances in Electrical and Computer Engineering, 17(2), 11-16. https://doi.org/10.4316/AECE.2017.02002

Wang, Y., \& Chi, Z. (2016). System of Wireless Temperature and Humidity Monitoring Based on Arduino Uno Platform. 2016 Sixth International Conference on Instrumentation \& Measurement, Computer, Communication and Control (IMCCC), 770-773. https://doi.org/10.1109/IMCCC.2016.89

Yilmaz, A. (2007). Sensor fusion in computer vision. 2007 Urban Remote Sensing Joint Event, URS, https://doi.org/10.1109/URS.2007.371863 